\title{
Synthesis and Fluorescence Properties of Some Aromatic Schiff Base Compounds
}

\author{
Heng Wang, Xin Qian* and Jie Li \\ College of Chemical Engineering and Materials, Zhejiang University of Technology, Hangzhou 310014, China \\ ${ }^{*}$ Corresponding author
}

\begin{abstract}
In this study, a series of aromatic aldehyde schiff base compounds were synthesized respectively by 4,4'diaminobiphenyl and three different aromatic aldehydes. The structures of these compounds were confirmed by FT-IR, and $1 \mathrm{H}$ NMR. The optical properties, which were characterized by UVVis and photoluminescence spectroscopy, indicated that the fluorescence spectra were red-shift with the increase of the electron delocalization in the conjugate systems. The result showed that these schiff base compounds might have potential application as fluorescence materials.
\end{abstract}

Keywords- schiff base; aromatic aldehyde; photoluminescence

\section{INTRODUCTION}

Schiff base are a kind of organic compounds with special group imine or azomethine(- $\mathrm{RC}=\mathrm{N}-)$ formed by condensation between aldehyde and primary amines.[1,2] Especially, due to the affection of the conjugation system, the aromatic aldehyde schiff bases show excellent photoluminescence properties[3,4]. Thus, these compounds might have great prospect in sensor, luminescence, eletroluminescent devices and biological[5-8]. In this study, three kinds of aromatic aldehydes were selected to regulate the affection of the conjugation system, which further influence the photoluminescence properties of these compounds.

\section{EXPERIMENTAL}

Synthesis of aromatic aldehydes schiff bases: The aromatic aldehydes schiff bases were obtained by a condensation reaction between 4,4'-diaminobiphenyl and three different aromatic aldehydes. The procedure was as follows: $4.2 \mathrm{mmol}$ aromatic aldehydes and $0.4 \mathrm{mmol}$ p-toluenesulfonic acid were dissolved in $20 \mathrm{ml}$ methanol with gently stirring under nitrogen atmosphere. After $2.0 \mathrm{mmol} 4,4^{\prime}$ '-diaminobiphenyl (DAP) was dispersed into $20 \mathrm{ml}$ methanol, the suspension was dropwised in the system. The reaction was kept at room temperature for 12h. Finally, three different yellow precipitates were obtained by filtration, and washed with ethanol twice.The pure products were dried in vacuum at room temperature until constant weight.

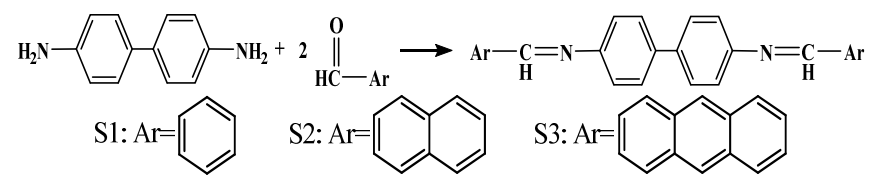

FIGURE I. SYNTHESIS OF THREE AROMATIC ALDEHYDE SCHIFF BASE COMPOUNDS

\section{RESULTS AND DISCUSSION}

As shown in Figure 1, three schiff bases have been characterized by Fourier Transform Infrared spectroscopy (FTIR). With the similar structures, the infrared spectra of the three compounds were also semblable. The peaks at $1617-1624 \mathrm{~cm}^{-1}$ were characteristically assigned to the carbon-nitrogen double band of the imine groups $(-\mathrm{HC}=\mathrm{N}-)$, while the peaks at 3354 $\mathrm{cm}-1$ and $3290 \mathrm{~cm}^{-1}$ were assigned to the C-H stretching vibration of the imine groups. Furthermore, the peaks at 3051 $\mathrm{cm}^{-1}$ were attributed to $\mathrm{C}-\mathrm{H}$ stretching vibration of aromatic ring, and the peaks around $1570 \mathrm{~cm}^{-1}$ and $1490 \mathrm{~cm}^{-1}$ that can be assigned to aromatic rings vibrations. All these demonstrated that the aromatic aldehyde schiff bases were synthesized successfully.

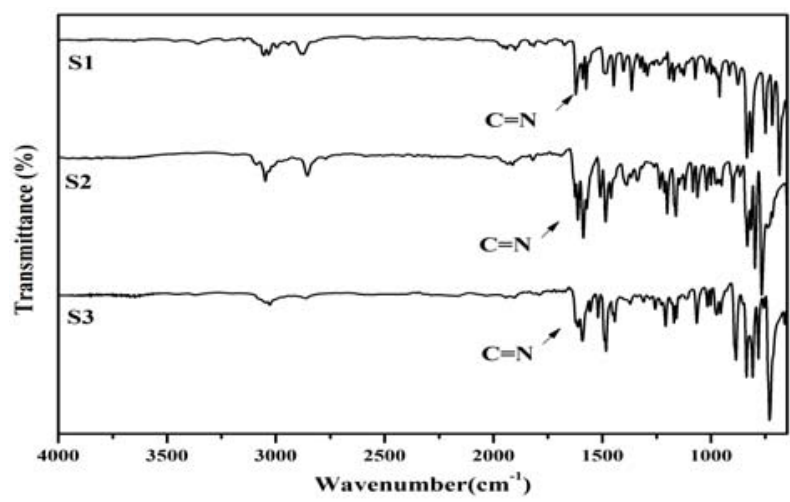

FIGURE II. FT-IR SPECTRA OF SCHIFF BASE S1,S2, S3

The chemical structures of three schiff bases were confirmed by ${ }^{1} \mathrm{H}$ NMR, further. As shown in Figure 2, all the resonance signals could be attributed to the protons of the aromatic aldehyde schiff bases. The typical $\mathrm{N}=\mathrm{C}-\mathrm{H}$ proton signals at 9.2-10.1 ppm was observed respectively in the spectra of S1, S2 and S3, due to the difference of the chemical surrounding of the conjugated systems.

To characterize the light absorption properties of the three different aromatic aldehyde schiff bases, the UV-Vis spectra were shown in Figure 3. Compared with the spectrum of 4,4'diaminabiphenyl (DAP), the detected peaks of the schiff bases was red-shift to $353 \mathrm{~nm}, 371 \mathrm{~nm}$ and $416 \mathrm{~nm}$. It indicated that the conjugated systems have obvious change, which meant the aromatic ring changed from benzene ring ( $\mathrm{S} 1)$, naphthalene ring (S2), to anthracene ring (S3), the conjugated systems became bigger and bigger. 


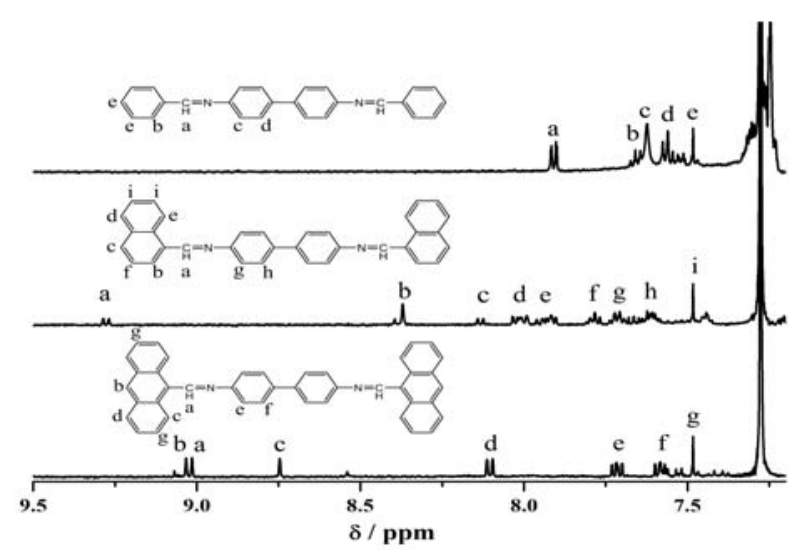

FIGURE III. ${ }^{1} \mathrm{H}$ NMR SPECTRA OF S1,S2,S3

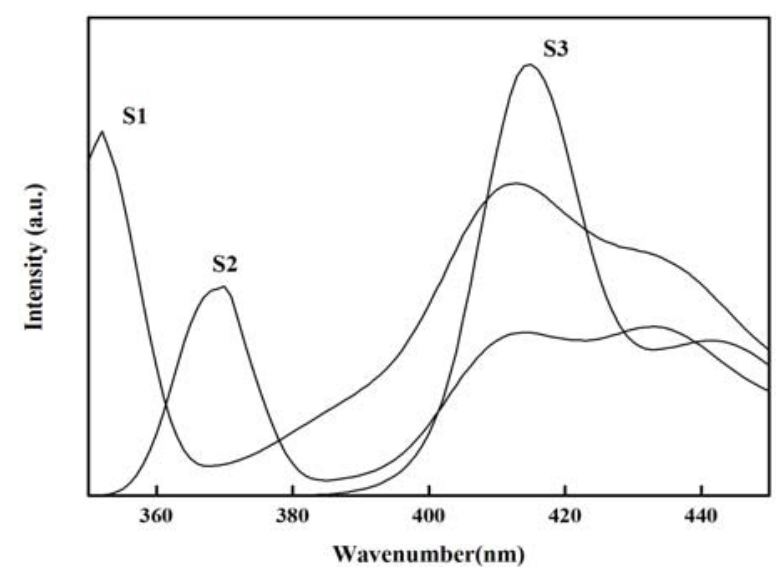

FIGURE IV. FLUORESCENCE EMISSION SPECTRA OF S1, S2,S3

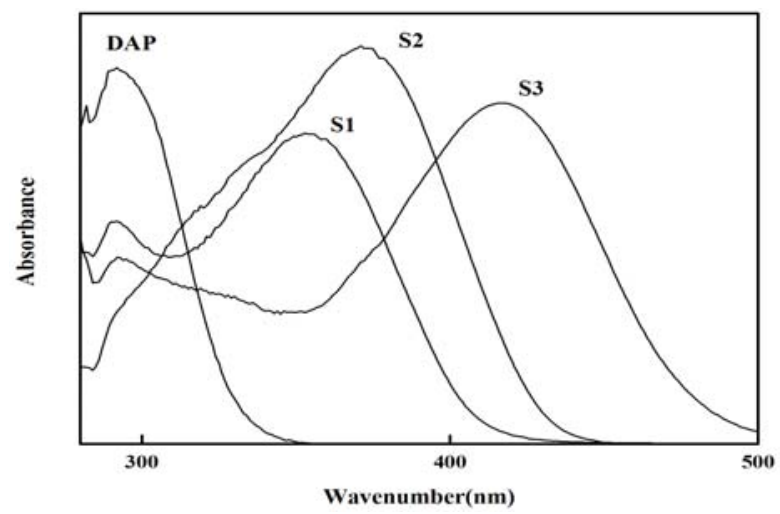

FIGURE V. UV-VIS ABSORPTION SPECTRA OF DPA,S1,S2,S3

The photoluminescence properties of the aromatic aldehyde schiff bases showed the similar phenomenon with the light absorption properties. The significant bathochromic shift was also because of the alteration of the conjugate systems with the different aromatic nucleuses.

\section{CONCLUSION}

A series of aromatic aldehyde schiff bases were successfully synthesized via simple condensation reaction. The structures of the compounds were confirmed by FT-IR and ${ }^{1} \mathrm{H}$
NMR, while the optical properties were characterized by UVVis and photoluminescence. The compounds showed the optical properties could be regulated by using different aromatic aldehydes as ingredient. These kinds of aromatic Schiff bases have a good potential application in luminescent materials.

\section{REFERENCES}

[1] Hosseini, M. G.; Ehteshamzadeh, M.; Shahrabi, T. Electrochimica Acta 2007, 52, (11), 3680-3685.

[2] Keleş, H.; Keleş, M.; Dehri, İ.; Serindağ, O. Materials Chemistry and Physics 2008, 112, (1), 173-179.

[3] Kumari, S.; Singh, A. K.; Rao, T. R. Materials Science and Engineering: C 2009, 29, (8), 2454-2458.

[4] Leçe, H. D.; Emregül, K. C.; Atakol, O. Corrosion Science 2008, 50, (5), 1460-1468.

[5] Li, S. H.; Chen, F. R.; Zhou, Y. F.; Wang, J. N.; Zhang, H.; Xu, J. G. Chem Commun (Camb) 2009, (28), 4179-4181.

[6] Pui, A.; Malutan, T.; Tataru, L.; Malutan, C.; Humelnicu, D.; Carja, G. Polyhedron 2011, 30, (12), 2127-2131.

[7] Shakya, P. R.; Singh, A. K.; Rao, T. R. Spectrochim Acta A Mol Biomol Spectrosc 2011, 79, (5), 1654-1659.

[8] Ziółek, M.; Sobczak, I. Journal of Inclusion Phenomena and Macrocyclic Chemistry 2008, 63, (3-4), 211-218.0. 